\title{
Bioassay-guided isolation and purification of phytochemicals with anti-parasitic properties from Cissus ruspolii
}

\author{
K. Tolossa ${ }^{1,2}$, S.C. Fry ${ }^{2}$, S. Athanasiadou ${ }^{3}$, G.J. Loake ${ }^{2}$ and J.G.M. Houdijk ${ }^{1}$ \\ ${ }^{1}$ Monogastric Science Research Centre, SRUC, Edinburgh EH9 3JG, UK, ${ }^{2}$ Institute of Molecular Plant Sciences, \\ University of Edinburgh, Edinburgh EH9 3BF, UK and ${ }^{3}$ Disease Systems, SRUC, Edinburgh EH9 3JG, UK
}

Many smallholder farmers and pastoralists rely on their indigenous knowledge, practices and locally available plants to manage both human and livestock parasitic infections ${ }^{(1)}$. We previously identified in screening study that aqueous crude extract and its relatively late-eluting fractions from one of such plants, Cissus ruspolii, possess strong in vitro anthelmintic properties ${ }^{(2,3)}$. Thus, these fractions may contain phytochemicals with anti-parasitic properties. The objective of this study was to further isolate, purify and characterise anthelmintic phytochemicals from C. ruspolii.

A crude aqueous extract of $C$. ruspolii was prepared by maceration in de-ionized water for $72 \mathrm{hr}$ at room temperature. This extract was fractionated on a Bio-Gel P-2 column chromatography using de-ionized water and fractions were bio-assayed in triplicate for egg hatch inhibition (EHI) using Teladorsagia circumcincta eggs as per guidelines for anthelmintic resistance testing ${ }^{(4)}$ to locate bio-active pools. Isolation and purification of phytochemicals from one of the active pools was carried out through Sephadex LH-20 column chromatography by solvent gradient elution, and were also bio-assayed in triplicate, using the chemical anthelmintic monepantel $\left(\right.$ Zolvix $^{\circledR} ; 0 \cdot 1 \mu \mathrm{g} / \mathrm{mL}$ ) as a positive control. Further characterization of compounds was done using thin-layer chromatography (TLC) developed in $\mathrm{BuOH}$ : $\mathrm{HOAc}: \mathrm{H}_{2} \mathrm{O}(4: 1: 1)$ and $\mathrm{CHCl}_{3}$ : $\mathrm{MeOH}$ : $\mathrm{HOAc}(10: 2: 1)$ solvent systems with markers, stained with vanillin- $\mathrm{H}_{2} \mathrm{SO}_{4}$ and heated in an oven at $120^{\circ} \mathrm{C}$ for 30 minutes.
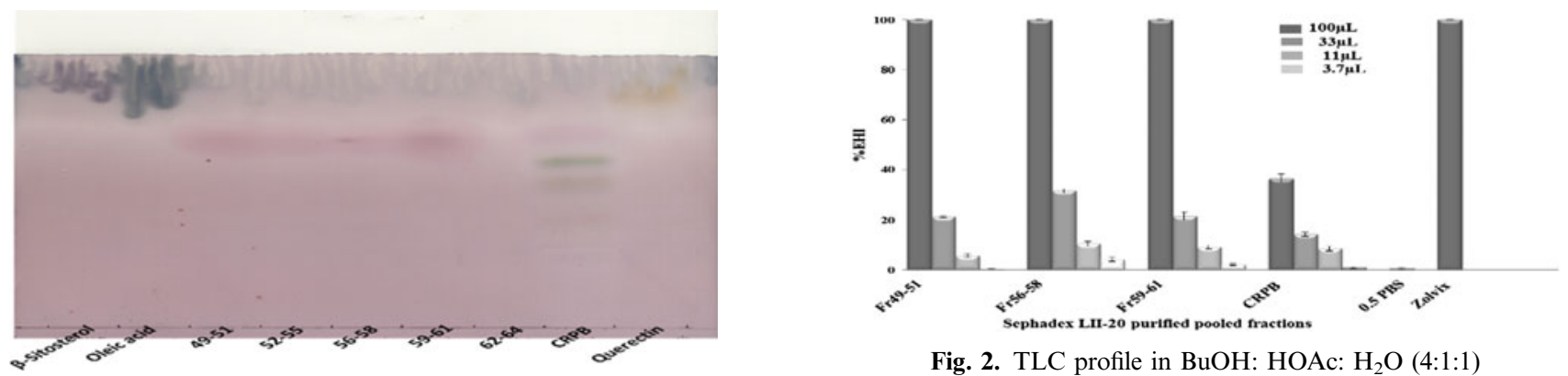

Fig. 1. EHI assays of purified C. ruspolii compounds

Fig. 2. TLC profile in BuOH: $\mathrm{HOAc:} \mathrm{H}_{2} \mathrm{O}(4: 1: 1)$

We observed highly significant and dose dependent EHI activities of three pools from the Bio-Gel P-2 fractionation (data not shown). The Sephadex LH-20 purified active compounds, which also showed strong and dose-dependent EHI activities (Figure 1); the highest concentration used for some of the components resulted in $100 \%$ EHI. The TLC analyses of these pure compounds indicated that they are polar compounds with higher average retention factor $\left(\mathrm{R}_{\mathrm{f}}=0.553\right)$ in polar and acidic solvent system (Figure 2$)$ than in relatively non-polar and acidic solvent system: chloroform- methanol-acetic acid $\left(\mathrm{R}_{\mathrm{f}}=0 \cdot 194\right.$; data not shown).

Our data support the view that $C$. ruspolii has anti-parasitic properties and also validated its ethno-medical use. Moreover, these in vitro experiments provided preliminary screenings for efficacy of extracts, fractions and purified compounds. Purified samples obtained have been submitted for NMR and MS analysis for structure elucidation, which is expected to inform pending in vivo trials, underpin existing phytotherapy and potentially the development of novel anthelmintic drugs.

The authors gratefully acknowledge funding from CIDLID, supported by DFID, BBSRC and SG (BB/H009299/1) and SRUC International Engagement Strategy.

1. Tolossa K et al. (2013) J Ethnobiol Ethnomed 9, 32

2. Tolossa K et al. (2015) Adv Anim Biosci 6, 92

3. Tolossa K et al. (2015) Adv Anim Biosci 6, 94

4. Coles GC et al. (2006) Vet Parasitol 136, 167-185 